\title{
A Numerical Method for Designing Three-Dimen- sional Wing Based on Surface Panel Method
}

\author{
by Yumin Su*, Member Mitsuhisa Ikehata*, Member \\ Hisashi Kai*, Member
}

\begin{abstract}
Summary
A numerical method for designing three-dimensional wing from the specified pressure distribution based on surface panel method is presented. A number of hyperboloidal quadrilateral panels are used to approximate the surface of an original wing and the hydrodynamic characteristics of this original wing are calculated by potential based surface panel method employing the pressure Kutta condition. Then the geometry of the original wing is made to change in a small amount and the pressure increment due to this change is calculated by the same method. From them the influence coefficient matrix of wing geometry on pressure distribution is constructed. Utilizing the difference between the specified pressure distribution and the pressure distribution of the original wing, a linear equation system about the geometrical corrector of wing is established. The original wing geometry is modified with this corrector to form a new wing for next iteration. By means of repeating this computation procedure the wing that will have the specified pressure distribution can be obtained.

Several wings have been designed as examples and the results have indicated that the method is effective to design the three-dimensional wing having the specified pressure distribution.
\end{abstract}

\section{Introduction}

For lifting body a great quantity of researches have been carried out and there are several kinds of theory for analysis and design of lifting body. Now by theoretical method various lifting bodies even including ones which have very complicated geometry such as marine propeller can be analysed satisfactorily.

On the other hand, many efforts also have been made to design a lifting body. Parkin \& Peebles ${ }^{1)}$ first derived a wing profile satisfying the given pressure distribution with conformal mapping technique. Eppler \& Shen ${ }^{2), 3)}$ designed a new series of wing operating at various angles of incidence by the same technique.

A stream function method for design of wing was presented by Ormsbee \& $\mathrm{Chen}^{4)}$ in 1972 . In this method the wing in two-dimensional potential flow is replaced by a vortex sheet on the wing surface. The relationship between stream function and vortex distribution is used to construct the integral equation and the geometry of wing is solved iteratively by this equation. Later on, modifications of this method were developed by Kennedy \& Marsden ${ }^{5)}$, Sonnie \& Laine $^{6)}$, and Dutt \& Sreekanth ${ }^{7}$.

Lee, Kim\&Suh ${ }^{8)}$ recently developed a surface panel

* Yokohama National University

Received 10th July 1997

Read at the Autumn meeting 14, 15th Nov. 1997 method for design of hydrofoil. They determine the total potential on an assumed hydrofoil from the specified pressure distribution. According to two ${ }^{-}$ dimensional Green's theorem they derive an integral equation that expresses the relationship between the total velocity potential and unknown local flux on hydrofoil surface. The geometrical corrector is calculated by integrating the local flux and new hydrofoil geometry is obtained by correcting assumed hydrofoil. Repeating this procedure, the hydrofoil corresponding to specified pressure distribution is designed.

All these methods mentioned above are based on two -dimensional theory so that there is the limitation for their application in some degree. Quite recently the authors have known that Bristow \& Hawk have developed a method for design of three-dimensional wing, but unfortunately we have no chance to read their paper.

In the present paper, a potential based surface panel method is used to calculate the pressure distribution of an original wing. A number of control points are taken for numerical calculation of design. All these control points are changed one by one and for each case the pressure increment is calculated to determine the influence coefficient matrix of wing geometry on pressure distribution. Using the specified pressure distribution a linear equation system is established and with it the geometrical corrector of the original wing is calculated. Consequently a new wing for next iteration can be obtained by modifying the original wing. To design 
the wing that has the specified pressure distribution, the iterative calculation should be carried out untill the difference between the specified pressure distribution and the pressure distribution of designed wing is small enough to be accepted.

Several three-dimensional wings are designed as examples for confirmation of capability of the present method.

\section{Description of design method}

\subsection{Surface panel method for analysis of wing}

We consider a wing in an inviscid, incompressible, and irrotational flow with an uniform velocity $\vec{V}_{A}$. A Cartesian coordinate system $\mathrm{O}^{-x y z}$ is defined as shown in Fig. 1. Origin $\mathrm{O}$ is fixed at midchord of medium section and $\mathrm{x}$ is measured along the chord of the wing (In present paper this coordinate system is always used except the case that the coordinate system is specially redefined.). In this case, the flow field perturbed by the wing can be characterized by a perturbation velocity potential $\phi$, which satisfies the Laplace equation

$$
\nabla^{2} \phi=0
$$

According to Green's identity, the perturbation potential at an arbitrary field point $\mathrm{P}(\mathrm{x}, \mathrm{y}, \mathrm{z})$ can be expressed as an integral on the boundary surface of flow field $S$, which is composed of wing surface $S_{B}$, wake surface $S_{W}$ and outer control surface $S_{\infty}$ that closes the wing and flow field:

$$
\begin{aligned}
4 \pi E \phi(P)= & \iint_{s}\left[\phi(Q) \frac{\partial}{\partial n_{Q}}\left(\frac{1}{R(P, Q)}\right)\right. \\
& \left.-\frac{\partial \phi(Q)}{\partial n_{Q}} \frac{1}{R(P, Q)}\right] d s
\end{aligned}
$$

where

$$
E= \begin{cases}0 & \text { for the point } P \text { inside } S \\ 1 / 2 & \text { for the point } P \text { on } \mathrm{S} \\ 1 & \text { for the point } P \text { outside } S\end{cases}
$$

$R(P, Q)=$ distance between field point $P(x, y, z)$ and boundary point $Q\left(x_{0}, y_{0}, z_{0}\right)$

$\frac{\partial}{\partial n_{Q}}=$ normal derivative to $S$ at point $\mathrm{Q}$

This perturbation potential $\phi$ should also satisfy the conditions as follows:

1) On the outer control surface $S_{\infty}$, the perturbation velocity due to the wing should vanish in the limit where the outer surface is an infinite distance from the body

$$
\nabla \phi \rightarrow 0, \quad \text { as } S_{\infty} \rightarrow \infty
$$

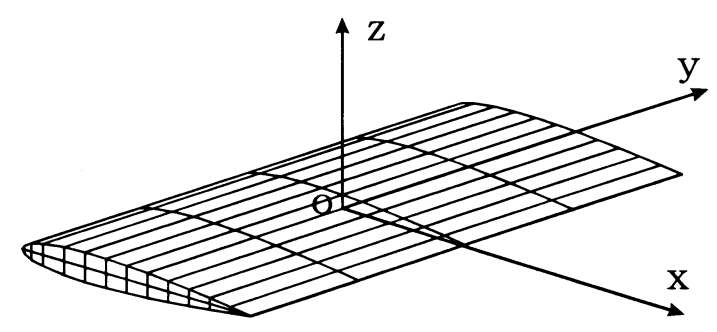

Fig. 1 Coordinate system of wing
2) On the wing surface, the kinematic boundary condition that normal velocity is zero should be satisfied.

$$
\frac{\partial \phi}{\partial n_{Q}}=-\vec{V}_{A} \cdot \vec{n}_{Q} \quad \text { on } S_{B}
$$

where $\vec{n}_{Q}$ is unit normal vector on the wing surface

3) We assume that the wake surface $S_{W}$ has zero thickness and there are not normal velocity jump and pressure jump across it, that is

$$
\begin{aligned}
& p^{+}-p^{-}=0 \\
& \left(\frac{\partial \phi}{\partial n_{Q_{1}}}\right)^{+}-\left(\frac{\partial \phi}{\partial n_{Q_{1}}}\right)^{-}=0 \quad \text { on } S_{W}
\end{aligned}
$$

here $Q_{1}$ is the point on the wake surface and superior + and - are used respectively to mark the values of upper and lower side of wing.

Considering above three conditions, on the boundary surface the integral equation (2) can be written as

$$
\begin{aligned}
2 \pi \phi(P)= & \iint_{S_{B}} \phi(Q) \frac{\partial}{\partial n_{Q}}\left(\frac{1}{R(P, Q)}\right) d s \\
& +\iint_{S_{W}} \Delta \phi\left(Q_{1}\right) \frac{\partial}{\partial n_{Q_{1}}}\left(\frac{1}{R\left(P, Q_{1}\right)}\right) d s \\
& +\iint_{S_{B}}\left(\vec{V}_{A} \cdot \vec{n}_{Q}\right) \frac{1}{R(P, Q)} d s \quad \text { on } S_{B}(6)
\end{aligned}
$$

where $\Delta \phi$ is the potential jump across the wake surface which can be expressed by

$$
\Delta \phi=\phi^{+}-\phi^{-} \text {on } S_{W}
$$

For the steady problem it is constant along a streamline in the wake.

For lifting problem a Kutta condition is needed at trailing edge to uniquely specify the circulation. In the present method the pressure Kutta condition is employed to determine the potential jump $\Delta \phi$ across the wake surface. This condition requires that the pressure difference between two sides of the wing at the trailing edge is zero, that is

$$
(\Delta p)_{T E}=p_{T E}^{+}-p_{T E}^{-}=0
$$

Integral equation ( 6 ) may be regarded as a representation of the perturbation velocity potential in terms of a normal doublet distribution of strength $-\phi(Q)$ on wing surface $S_{B}$, a normal doublet distribution of strength $-\Delta \phi\left(Q_{1}\right)$ on wake surface $S_{W}$, and a source distribution of strength $-\left(\vec{V}_{A} \cdot \vec{n}_{Q}\right)$ on $S_{B}$. This is a Fredholm integral equation of the second kind for the unknown doublet strength which equals potential value on wing surface.

Employing the pressure Kutta condition, the integral equation (6) can be uniquely solved by means of numerical iterative method.

The velocities and the pressure distributions on the wing surface can be determined by differentiating the velocity potential over the wing surface and by apply. ing Bernoulli's theorem.

\subsection{Design of wing}

At first we take a wing as the original wing of design, which has the same plan form as the target wing and arbitrary sections. Using the analysis method presented above the hydrodynamic characteristics of the original wing are calculated. Generally the pressure distribution 
of the original wing $C_{p}^{1}$ is different from the specified pressure distribution $C_{p 0}$ and the difference between them is noted as $\Delta C_{p}$. In order to determine the influence coefficient of wing geometry on the pressure distribution, we give geometry of the original wing a small increment $\delta z$ at one point and calculate corresponding increments of the pressure distribution $\delta C_{p}^{1}$ with the same surface panel method. Then the influence coefficient of this point can be calculaed by

$$
A=\frac{\delta C_{p}^{1}}{\delta z}
$$

After computing the influence coefficients of all points on the wing surface by the same way, a Jacobian matrix of the influence coefficient of the wing geometry on the pressure distribution is constructed. Consequently, a linear equation system about unknown geometrical corrector can be established as follows :

$$
[A][\Delta z]=\left[\Delta C_{p}\right]
$$

where

$[A]=$ Jacobian matrix of the influence coefficient

$[\Delta z]=$ matrix of the geometrical corrector of the wing

$\left[\Delta C_{p}\right]=$ matrix of the pressure difference between $C_{p 0}$ and $C_{p}^{1}$

By above equation, the geometrical correctors are determined from the pressure difference $\Delta C_{p}$. Finally we modify the original wing with these geometrical correctors to form a new wing that is nearer the target wing than the original wing. The new wing will be used as an original wing for next iterative computation. Repeating whole calculating procedure we can make the pressure distribution of the original wing comes near to the specified pressure distribution step by step untill the difference of them becomes small enough and thus ultimately the designed wing can be obtained.

\section{Numerical procedure}

For lifting body the wake surfaces always exist in the downstream behind it. Usually we do not know the exact form of the wake surfaces. In the present method the wake surface of the wing is assumed as a plane wake surface on the same plane which the chord surface of the wing is on.

In order to obtain a numerical solution of the integral equation ( 6 ), the wing surface and the wake surface are divided into a number of small elements. Each element is approximated by a hyperboloidal quadrilateral panel. The numbers of the panels on the wing surface and the wake surface are respectively $N$ and $N_{R}$. Perturbation potential $\phi$, potential jump $\Delta \phi$ and $\left(\vec{V}_{A} \cdot \vec{n}\right)$ are all assumed to be constant within each panel, so that boundary integral equation ( 6 ) can be written as a linear equation system

$$
\begin{aligned}
& \sum_{j=1}^{N}\left(\delta_{i j}-C_{i j}\right) \phi_{j}-\sum_{k=1}^{N_{R}} W_{i k} \Delta \phi_{k}= \\
& \quad-\sum_{j=1}^{N} B_{i j}\left(\vec{V}_{A} \cdot \vec{n}_{j}\right) \quad i=1,2, \ldots, N
\end{aligned}
$$

here $\delta_{i j}$ is the Kronecker delta and $C_{i j}, W_{i k}$ and $B_{i j}$ are influence coefficients defined by

$$
\begin{aligned}
C_{i j} & =\frac{1}{2 \pi} \iint_{s_{j}} \frac{\partial}{\partial n_{j}}\left(\frac{1}{R_{i j}}\right) d s_{j} \\
W_{i k} & =\frac{1}{2 \pi} \iint_{s_{k}} \frac{\partial}{\partial n_{k}}\left(\frac{1}{R_{i k}}\right) d s_{k} \\
B_{i j} & =\frac{-1}{2 \pi} \iint_{s_{j}}\left(\frac{1}{R_{i j}}\right) d s_{j}
\end{aligned}
$$

where $S_{j}$ and $S_{k}$ are the surface panels on the wing surface and the wake surface. $R_{i j}$ and $R_{i k}$ are the distances from the boundary point on $i$-th panel to integration points on $S_{j}$ and $S_{k}$.

All these influence coefficients $C_{i j}, W_{i k}$ and $B_{i j}$ are calculated by Morino's analytical formulation ${ }^{9}$. Thus the linear equation system (11) can be solved numerically to obtain the values of perturbation potential $\phi_{j}$ on the wing surface.

The velocities and the pressure distributions on the wing surface are calculated with the same numerical method as which Hoshino used ${ }^{10}$.

For the numerical calculation of design, a number of control points are selected on the wing surface. $z$ direction coordinates of control points are expressed by $z_{m}$

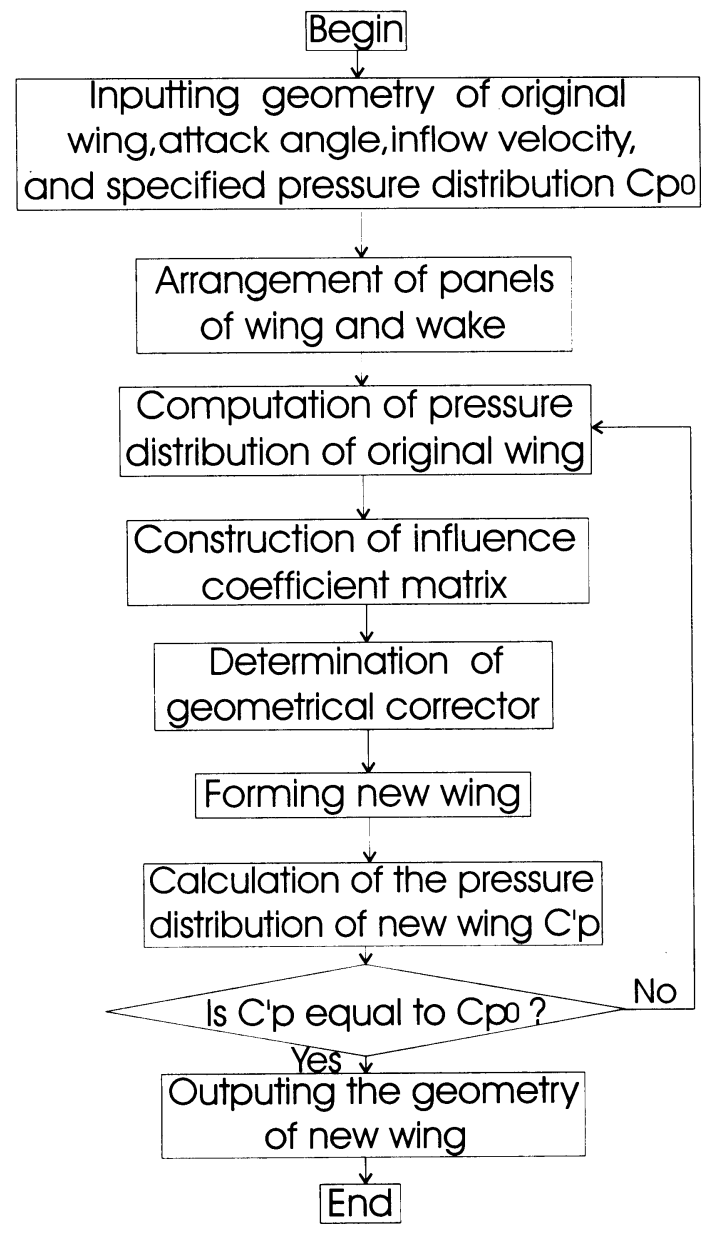

Fig. 2 Flow chart of design calculation of wing 
( $m$ is the number of a control point) and corresponding to them, the specified pressure distribution is expressed by $C_{p 0 m}$ and the pressure distribution of the original wing is expressed by $C_{p m}^{1}$. Then equations ( 9 ) and (10) can be written as

$$
\begin{aligned}
& A_{l_{m}}=\frac{\delta C_{p l}^{1}}{\delta z_{m}} \\
& \sum_{m=1}^{N_{m}} A_{l m} \Delta z_{m}=\Delta C_{p l} \quad l=1,2, \ldots, N_{m}
\end{aligned}
$$

where

$A_{l_{m}}=$ influence coefficient induced by geometrical increment of $m$-th point on the pressure of $l$-th point

$\Delta z_{m}=$ geometrical corrector of $m$-th control point

$\Delta C_{p l}=$ pressure difference between $C_{p 0}$ and $C_{p}^{1}$ at $l$-th control point

$N_{m}=$ number of control points to be selected

The numerical calculation is carried out following the flow chart shown in Fig. 2. The iterative procedure is repeated untill the largest difference between the pressure distribution of the designed wing and the specified pressure distribution is smaller than a given precision value. At the same time the original wing is gradually modified to the designed wing that is expected.

\section{Examples of numerical design}

\section{1 About the specified pressure distribution}

In order to confirm the applicablity of the program of the surface panel method in the present design method, an ellipsoid is selected as an example to analyse. The ellipsoid is at zero attack angle and has semi-lengths of three axes given as $a=b=1$ and $c=0.1$. It is approximated by $800(20 \times 20 \times 2)$ panels shown in Fig. 3 . As shown in Fig. 4 the present calculation results have a good agreement with theoretical solution ${ }^{11}$. It indicates that the program of the surface panel method utilized in the present method is effective for analysis of three-

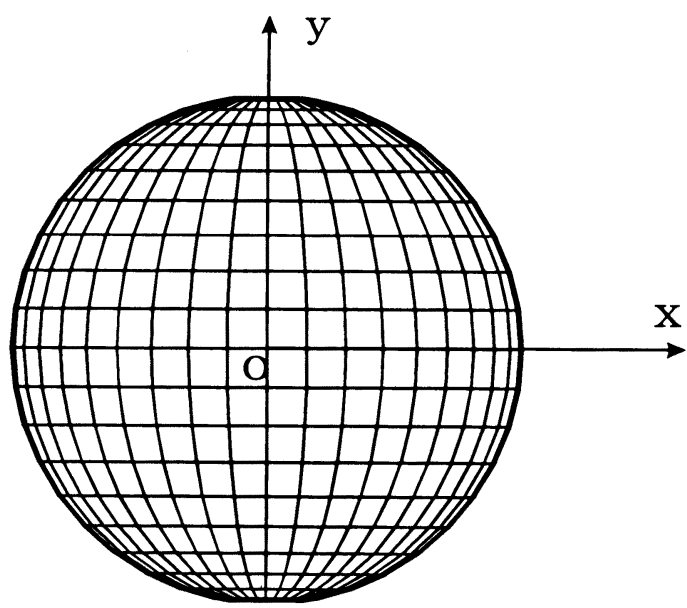

Fig. 3 Coordinate and panel arrangement of ellipsoid dimensional wing.

In the present method the specified pressure distribution has to be given as the condition of design. Because we use the surface panel method for calculating influence coefficient, if the original wing surface is not a smooth surface, the matrix of influence coefficient will become singular. And after the first iteration the original wing is dependent on the specified pressure distribution by equation (14). If the specified pressure distribution has a jump at some point, the matrix of the influence coefficient will be easy to become singular and in this case iterative calculation will not be convergent. Therefore, the specified pressure distribution should be a continuous curved surface. For following examples the specified pressure distributions are calculated by the
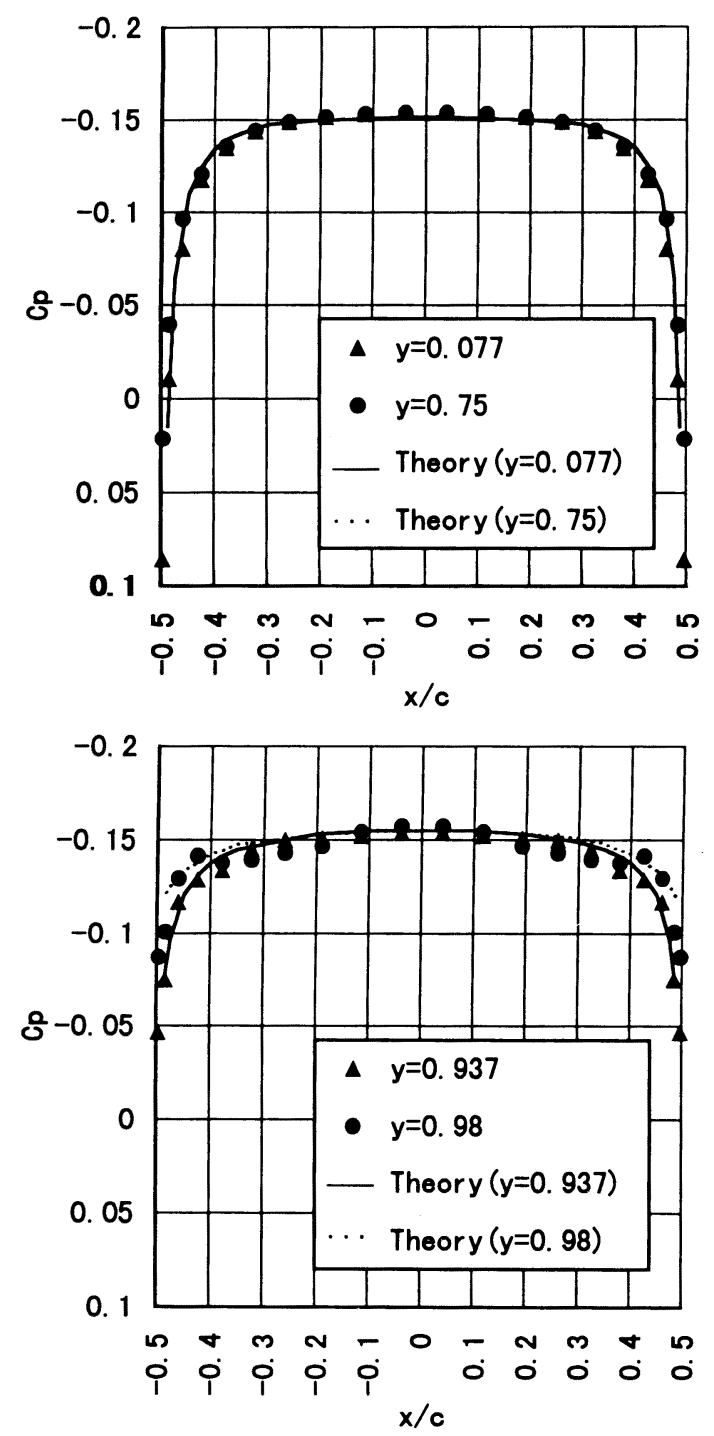

Fig. 4 Comparison of present calculation and theory results $^{11}$ for ellipsoid 
surface panel method described in this paper except the flattop pressure distribution.

\section{2 Rectangular wing with same sections in the spanwise direction}

First example is a rectangular wing which has the same sections in the spanwise direction. The aspect ratio of the target wing is 2.0 and its attack angle is 4 . 0 degree. The section of the target wing is nonsymmetrical NACA 4412 section. A wing that has same plan form and the same attack angle as target wing but has NACA 0010 section is taken as the original wing. As shown in Figs. 5 and 6, after twelve iterations the largest pressure difference between the designed wing and the target wing becomes smaller than five percent of the specified pressure. At the same time, the designed wing becomes very near the target wing.

In Figs. 7 and 8, designed results of another example that has same plan form but has NACA $66 \bmod +\mathrm{a} 0.8$ section are shown. The thickness ratio of this example is eight percent and camber ratio is five percent. This kind of section has well-distributed pressure distribution so that it is often used for marine propellers to avoid cavitation. The attack angle is also 4.0 dgree. The results indicate that the designed wing and its pressure distribution have a satisfactory agreement with the target wing and the specified pressure distribution.

In Fig. 5 through Fig. 8, we can also see that although the pressure distributions are almost overlapping each other there is still a little difference between the designed wing and the target wing near the trailing

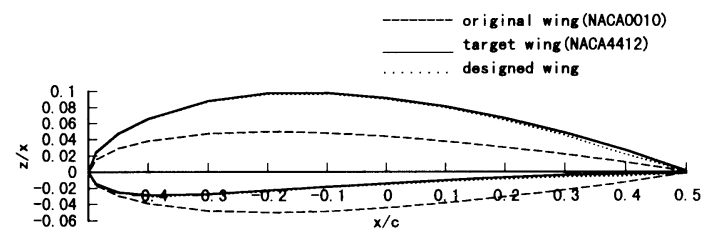

Fig. 5 Designed rectangular wing for target wing with NACA4412 section

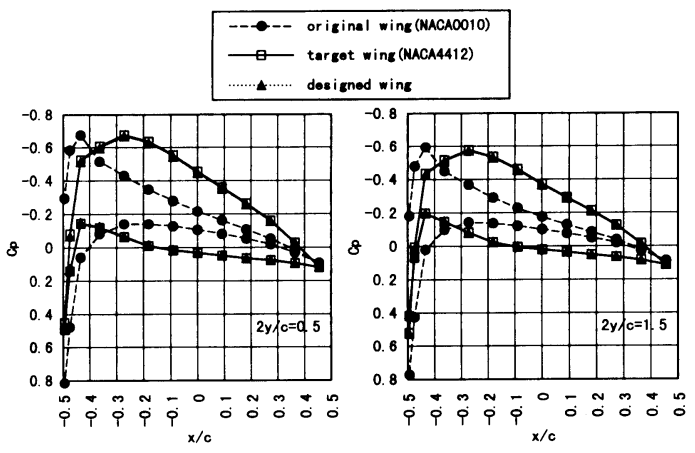

Fig. 6 Pressure distribution of designed rectangular wing for target wing with NACA4412 section edge (Figs. 5 and 7) and on the suction surface (Fig. 7). Such results are considered to be due to the number of panels and the arrangements of both panels and control points.

4. 3 Rectangular wing with continuously varying thickness and camber in the spanwise direction

Next example is the rectangular wing which chords are same but thickness and camber continuously vary in the spanwise direction. The coordinate system and the panel arrangement are shown in Fig. 9. The thickness ratio changes linearly from 12 percent of one end to 4 . 8 percent of other end and the camber ratio changes linearly from 4 percent to 1.6 percent. The attack angle is zero. The calculation of design is carried out with an original wing with symmetrical sections.

In Figs. 10 and 11, it can be seen that although the sections and corresponding pressure distributions are

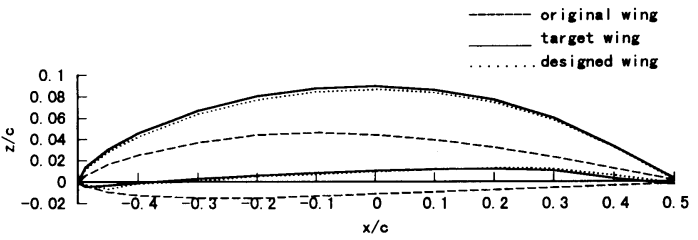

Fig. 7 Designed rectangular wing for target wing with NACA $66 \bmod +\mathrm{a} 0.8$ section

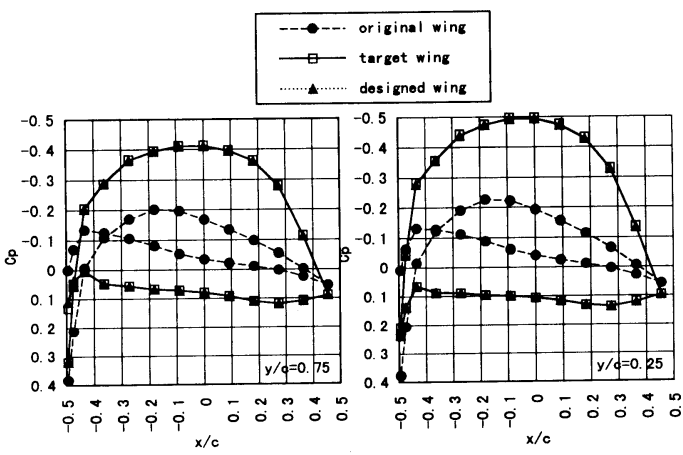

Fig. 8 Pressure distribution of designed rectangular wing for target wing with NACA $66 \bmod +\mathrm{a} 0.8$ section

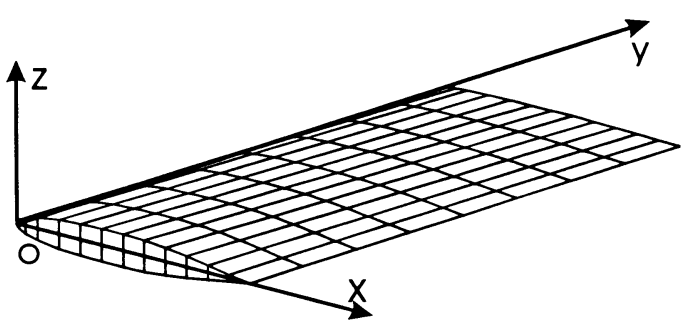

Fig. 9 Coordinate of rectangular wing 


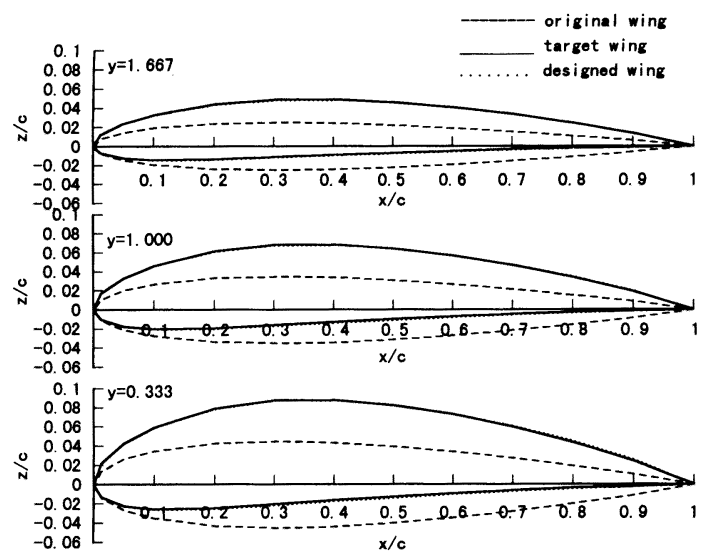

Fig. 10 Designed rectangular wing with different thickness and camber in spanwise direction
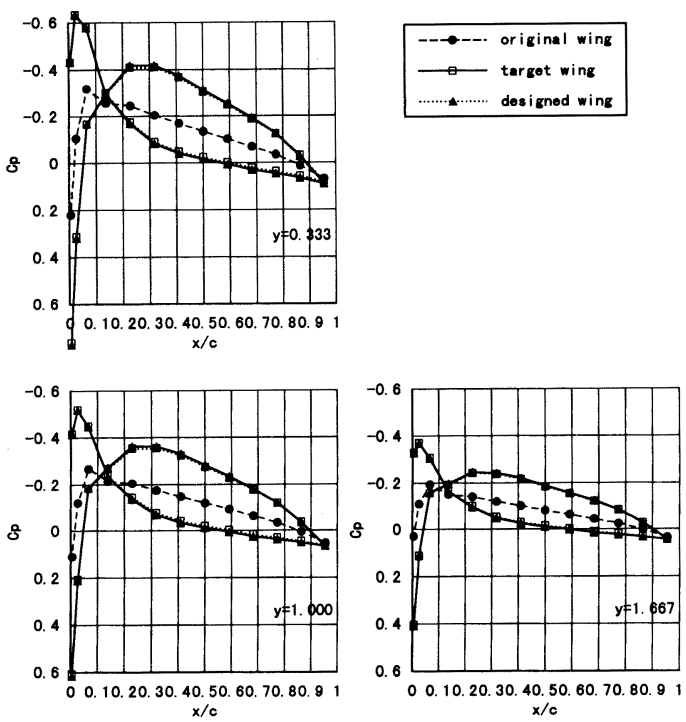

Fig. 11 Pressure distribution of designed rectangular wing with different thickness and camber in spanwise direction

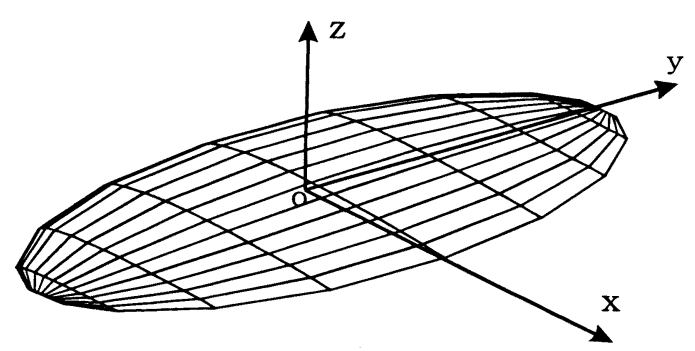

Fig. 12 Coordinate and panel arrangement of elliptical wing quite different in the spanwise direction, when the pressure differences between the target wing and the designed wing become smaller than 5 percent for every sections, there are no significant geometrical differences between the designed wing and the target wing.

\section{4 Elliptical wing}

An elliptical wing with symmetrical sections also is designed as an example. The elliptical wing has semilengths of two axes given as $a=0.5$ and $b=1.0$. Its thickness distribution is parabolic which thickness ratio is 24 percent at mid-span and zero at tip. The attack angle is zero too. The coordinate system and the panel arrangement of the elliptical wing are shown in Fig. 12.

In Figs. 13 and 14 we can see that begining with the original wing that has the same elliptical plan form and 10 percent largest thickness ratio, after fourteen iterations the designed wing and its pressure distribution still have about 10 percent difference with the target wing and the specified pressure distribution, and after nineteen iterations the designed wing and its pressure distribution become indistinguishable from the target wing and the specified pressure distribution.

\subsection{Flattop pressure distribution}

In order to make the load distribution of the wing be uniform in the chordwise direction to avoid the generation of cavitation on the wing surface, a flattop pressure distribution of the wing as shown in Fig. 15 is often required in many situations. As the final example we try to design a rectangular wing having the flattop pressure distribution. The designed wing is assumed that it has same sections in the spanwise direction and its aspect ratio is 2.0. The attack angle is taken to be 4.0 degree. Since the pressure coefficient of wing in potential flow has a tendency to increase to 1.0 at

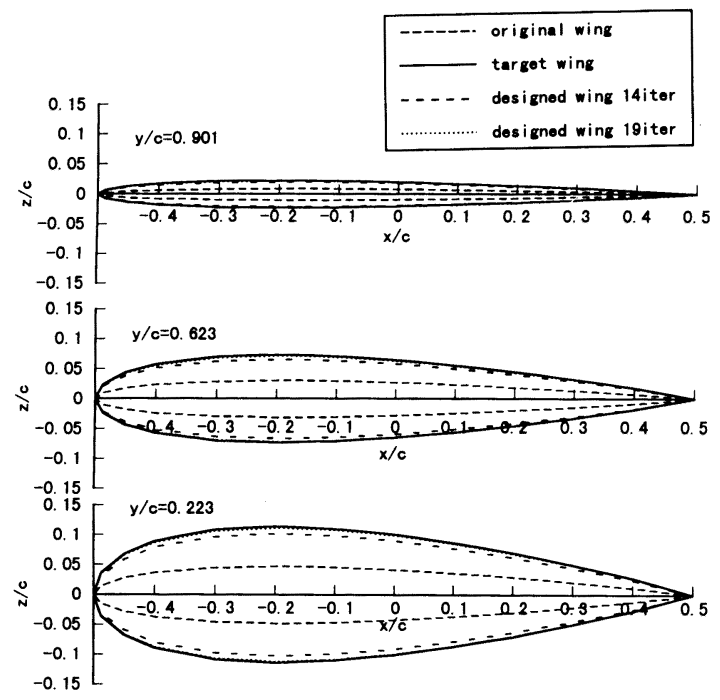

Fig. 13 Designed elliptical wing for target wing with 24 percent largest thickness 
leading edge, in the present research the pressure distribution shown in Fig. 16 is selected as the specified pressure distribution. We also take the wing with NACA 0010 section as the original wing.

In Fig. 16 it is shown that after sixteen iterations the difference between the pressure distribution of the designed wing and the specified pressure distribution becomes smaller than 5 percent of the specified pressure distribution. Corresponding to it, the designed wing
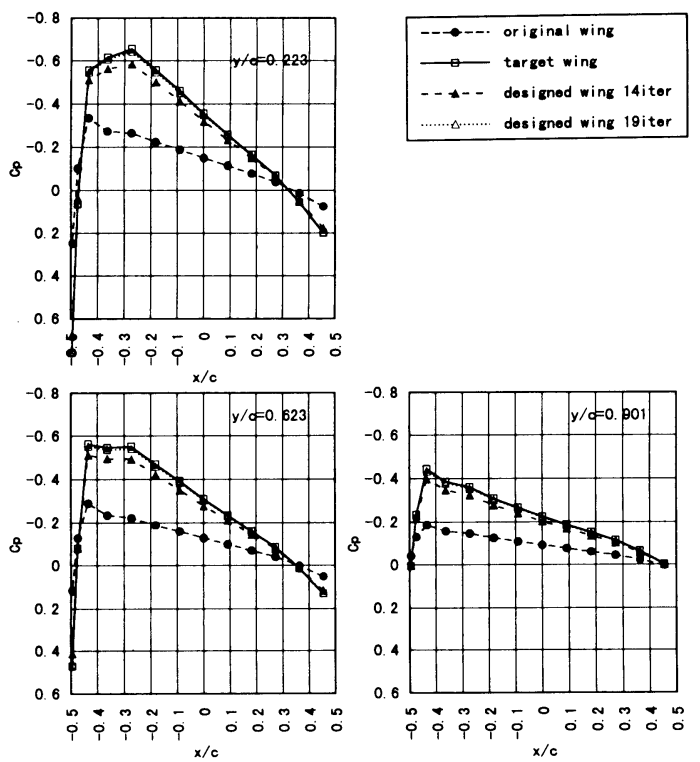

Fig. 14 Pressure distribution of designed elliptical wing for target wing with 24 percent largest thickness

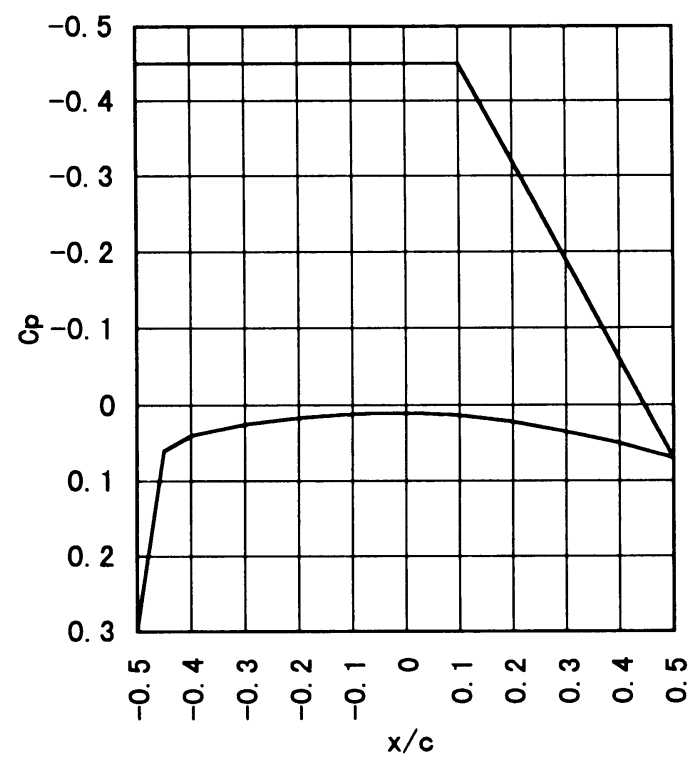

Fig. 15 Flattop pressure distribution shown in Fig. 17 is obtained. Evidently the pressure surface of the designed wing is not smooth enough for engineering use. That might be because the specified pressure distribution is selected arbitrarily and it probably do not suit for a smooth wing. The numbers and arrangements of panels and control points are considered to affect designed results too. Therefore, in order to obtain a smooth designed wing, the pressure distribution of the target wing should be specified very carefully.

For overcoming this problem the geometry of designed wing can be smoothed in a small quantity. In general case its pressure distribution will be just changed a little. For this example the designed wing is smoothed as shown in Fig. 18. Its pressure distribution
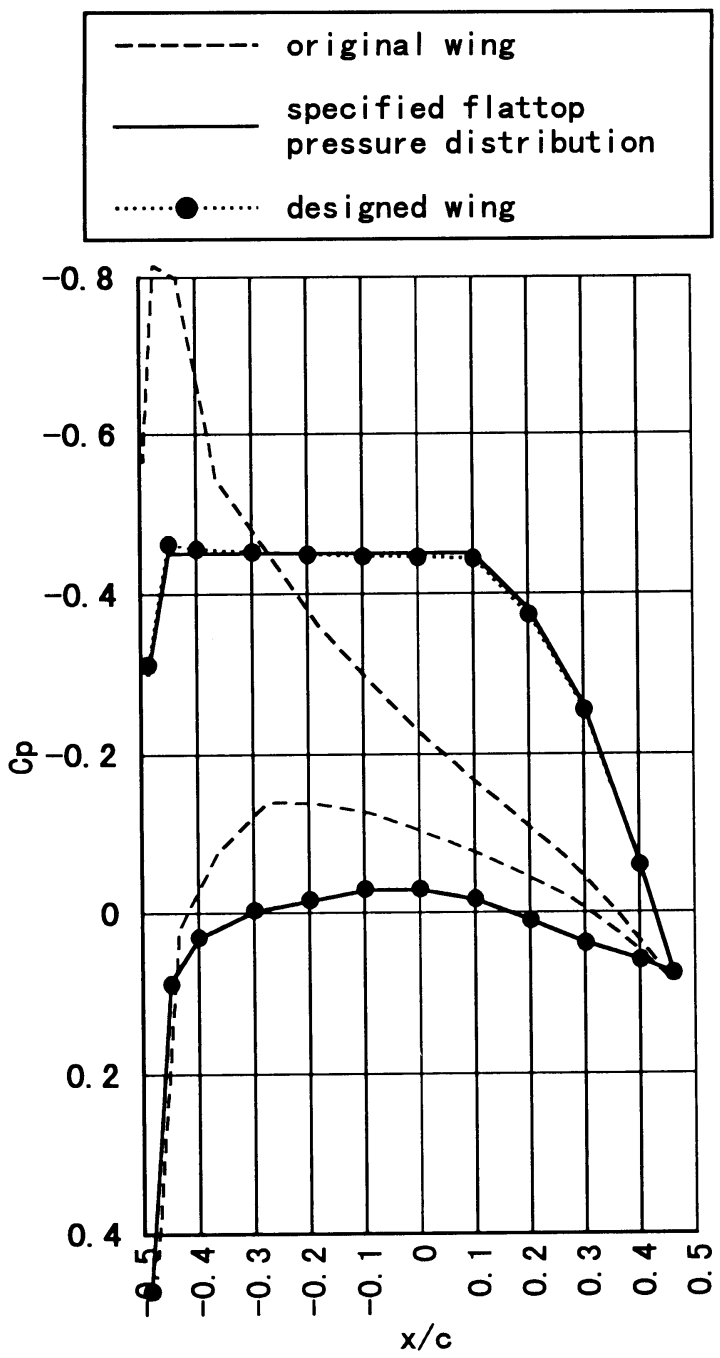

Fig. 16 Comparison of specified flattop pressure distribution and pressure distribution of designed wing 


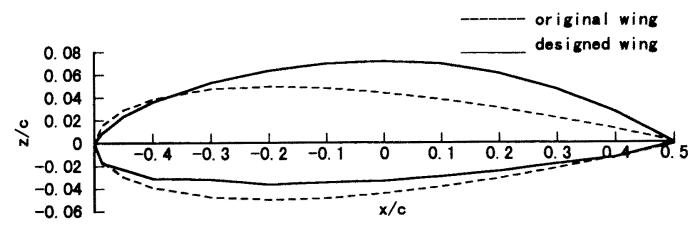

Fig. 17 Designed wing for flattop pressure distribution

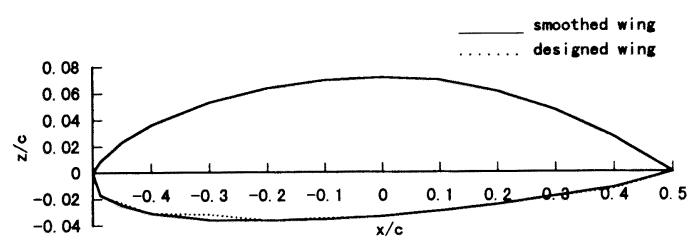

Fig. 18 Smoothed wing

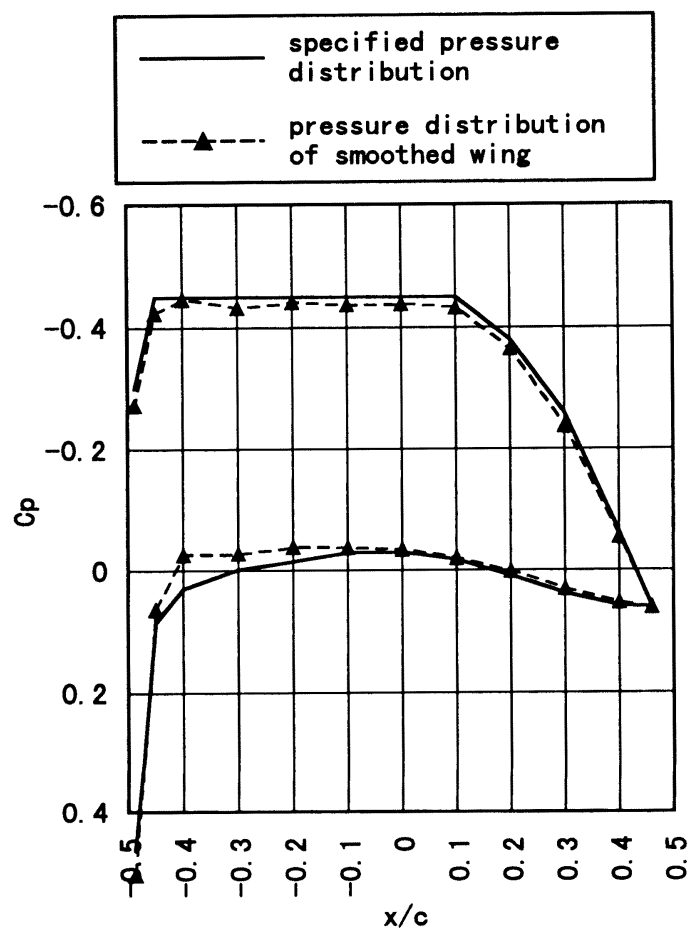

Fig. 19 Pressure distribution of smoothed designed wing

shown in Fig. 19 only has quite small changes and is still close to the specified pressure distribution.

\section{Conclusions}

A numerical method for designing the three-dimensional wing having the specified pressure distribution has been presented. The potential based surface panel method for three-dimensional lifting body, in which the pressure Kutta condition is employed, is directly applied for the analysis of the original wing and the determination of the influence coefficient matrix of the wing geometry on the pressure distribution. With this matrix a linear equation system is established to relate the geometry of the wing to the specified pressure distribution. Since there is no any hypothesis for the wing geometry in the present method, it could be expected that the present method can be applied for designing any kind of wings. The results of design for example wings indicated that the present method is effective for designing the three-dimensional wing having the specified pressure distribution.

\section{Acknowledgments}

The authors would like to thank Associate Professor K. Suzuki of Department of Naval Architecture and Ocean Engineering, Yokohama National University, for his useful discussions and suggestions during this research. Thanks are also due to Mr. I. Okada, technical assistant of YNU for his help and to all student friends of the hydrodynamics laboratory of YNU for their discussions.

\section{References}

1) Parkin, B. R. and Peebles, B. H. : Calculation of Hydrofoil Sections for Prescribed Pressure Distributions, SNAME Technical and Research Bulletin No. 1-17, 1956.

2) Eppler, R. and Shen, Y. T.: Wing Sections for Hydrofoils-Part 1: Symmetrical Profiles, Journal of Ship Research, Vol. 23, Sept. 1979, pp. 209217.

3) Eppler, R. and Shen, Y. T.: Wing Sections for Hydrofoils-Part 2: Nonsymmetrical Profiles, Journal of Ship Research, Vol.25, Sept. 1981, pp 191-200.

4) Ormsbee, A. I. and Chen, A. W.: Multiple Element Airfoils Optimized for Maximum Lift Coefficient, AIAA Journal, Vol. 10, Dec. 1972, pp. 1620-1624.

5) Kennedy, J. L. and Marsden, D. J. : A Potential Flow Design Method for Multicomponent Airfoil Sections, Journal of Aircraft, Vol. 15, Jan. 1978, pp. 47-52.

6) Soinne, E. and Laine, S.: An Inverse Boundary Element Method for Single Component Airfoil Design, Journal of Aircraft, Vol. 22, Jun. 1985, pp. 541-543.

7) Dutt, H. N. V. and Sreekanth, A. K.: Design of Aerofoils for Prescribed Pressure Distribution in Viscous Incompressible flows, Aeronautical Quarterly, Feb. 1980, pp. 42-55.

8) Lee, C. S. and Kim, Y. G. and Suh, J. C.: A Surface Panel Method for Design of Hydrofoil, Journal of Ship Research, Vol. 38, Sept. 1994, pp. 157181.

9) Morino, L. and Chen, L. T. and Suciu, E. O.: Steady and Oscillatory Subsonic and Supersonic Aerodynamics around Complex Configurations, AIAA Journal, Vol. 13, Mar. 1975, pp. 368-374.

10) Hoshino, T.: Hydrodynamic Analysis of Propellers in Steady Flow Using a Surface Panel 
Method, Journal of The Society of Naval Architects of Japan, Vol. 165, 1989, pp. 55-70.

11) Lee, J. T. : A Potential Based Panel Method for the Analysis of Marine Propellers in Steady Flow, Ph. D thesis, Dept. of Ocean Engineering, MIT, 1987. 